\title{
Ideologische Integration, massenmediale Täuschung: Zur Rolle von Propaganda in liberalen Demokratien
}

\author{
Florian Zollmann
}

Keywords: Propaganda, Massenmedien, Wissenschaft, Demokratie, Ideologie

\begin{abstract}
Übergeordnetes Ziel dieses Textes ist es, auf die zentrale Bedeutung von Propaganda in liberalen Demokratien hinzuweisen. Insbesondere frühe Forscherinnen und Forscher hatten auf die Rolle von Propaganda zur Herstellung von Konsens sowie zur sozialen und ideologischen Kontrolle hingewiesen. Zunächst wird daher anhand einer kritischen Diskussion früher und gegenwärtiger Forschungsarbeiten gezeigt, wie eine selektive Betrachtungsweise in der Wissenschaft und terminologische Veränderungen zu einer Marginalisierung des Forschungsgegenstandes Propaganda in liberalen Demokratien führten. Anschließend werden vernachlässigte Konzepte herausgearbeitet, die es ermöglichen, die Manifestation von Propaganda in den Massenmedien zu untersuchen. Um Propaganda sichtbar zu machen, wird ideologische Integration als eine Dimension von Propaganda konzeptualisiert und anhand kurzer Beispiele skizziert.
\end{abstract}

Zollmann, Florian. 2019. „Ideologische Integration, massenmediale Täuschung: Zur Rolle von Propaganda in liberalen Demokratien“. In Ideologie, Kritik, Öffentlichkeit. Verhandlungen des Netzwerks Kritische Kommunikationswissenschaft, herausgegeben von Uwe Krüger und Sebastian Sevignani, 228-252. Universität Leipzig. DOI: $10.36730 /$ ideologiekritik.2019.11

Florian Zollmann | Newcastle University | florian.zollmann@newcastle.ac.uk 


\section{Einführung}

Propagandastudien haben eine lange Tradition in den Medien- und Kommunikationswissenschaften und beschäftigten schon Gründungsväter des Feldes wie Harold D. Lasswell (vgl. 1971 [1927]). Die in den letzten Jahren aufgekommene Diskussion über sogenannte "Fake News" und Desinformation hat wieder zu einer vermehrten Auseinandersetzung mit dem Thema Propaganda geführt. Allerdings richtet sich der Fokus vieler Studien auf zentralisierte Propaganda autoritärer Staaten oder auf neuere Formen der Meinungsmanipulation, ausgeführt von einzelnen Akteuren mittels verschiedener (Sozial-)Medien. Der Mainstream der Medien- und Kommunikationswissenschaften postuliert die Sichtweise, in westlichen Staaten würden Beeinflussungstechniken größtenteils mit wohlwollendenden Intentionen, zur Informationsvermittlung, Überredung oder Überzeugung eingesetzt (vgl. Bakir et al. 2019; Dudley 1947, 107; Merten 2000, 151).

Diese Forschungsausrichtung spiegelt kaum die Bedeutung von Propaganda wider. Drew Dudley $(1947,107)$, Leiter der Medienabteilung des US-amerikanischen Office of War Mobilization and Reconversion nach dem Zweiten Weltkrieg, bemerkte: „Actually, Hitler did employ the technique of advertising during the prewar and war years, frequently referring to America's advertising in glowing and admiring terms in Mein Kampf, and later utilizing advertising's powerful repetitive force to the utmost [Hervorhebung im Original]." Wie dieses Zitat und andere Forschungsarbeiten belegen, ist moderne Propaganda von der Werbe- und PublicRelations-Industrie in liberalen Demokratien mit einer Vorreiterrolle der USA entwickelt worden (vgl. Carey 1995, 14). Zahlreiche Akteure inklusive der Nationalsozialisten bedienten sich dieser manipulativen Techniken, deren Ausmaß sich in den vergangenen Jahrzehnten vergrößert hat (vgl. Miller und Dinan 2008, 5-18; Miller und Robinson 2019). Auch fungieren die Massenmedien als wichtige Institution zur Verbreitung von Propaganda (vgl. Zollmann 2019). Denn insbesondere in liberalen Demokratien stellt die Schaffung und Aufrechterhaltung von emotional „signifikanten Symbolen" mit dem Ziel, die Sonderinteressen von Politik- und Wirtschaftseliten sowie von Großkonzernen gegenüber populären politischen Strömungen und der Arbeiterbewegung zu verteidigen, ein weitreichendes Phänomen dar (Carey 1995, 14, 19). 
Dieser Beitrag soll ein Versuch sein, das vorherrschende Bild, das Propaganda eher ein historisches Phänomen sei, lediglich auf Sonderfälle zutreffe oder nur in autoritären Staaten zur Geltung komme, zurechtzurücken.

Es wird zunächst aufgezeigt, wie nach dem Zweiten Weltkrieg eine Politisierung der Forschung zu einer selektiven Anwendung des Propagandakonzeptes in Wissenschaft und Gesellschaft führte. ${ }^{1}$ Denn die Tradition einer "Kritischen Theorie" blühte in Deutschland lediglich in den 1960er und 1970er Jahren noch einmal auf (vgl. Scheu und Wiedemann 2008). Die Medien- und Kommunikationswissenschaften zeichnen sich seither vor allem durch ein angepasst stromlinienförmiges und machthöriges Forschungsprogramm aus. Anschließend werden vernachlässigte Konzepte herausgearbeitet, die es ermöglichen, Propaganda in den Massenmedien liberalen Demokratien zu untersuchen. Darauf aufbauend wird Integrationspropaganda, die eine Dimensionen massenmedial verbreiteter Propaganda beschreibt, mit Verweis auf Fachliteratur ausdifferenziert. Zur weiteren Veranschaulichung werden konkrete Beispiele hinzugezogen. Übergeordnetes Ziel ist es, einen Werkzeugkasten der Propagandaforschung zu etablieren (vgl. Silverstein 1987, 50, 54). Dies geschieht in der Hoffnung, dass andere Forscherinnen und Forscher sich ebenfalls kritisch mit dem Thema Propaganda in liberalen Demokratien auseinandersetzen werden. ${ }^{2}$

$1 \quad$ Mit Politisierung ist hier gemeint, dass wissenschaftliche Forschung die gleichen ideologischen Grenzziehungen vornimmt wie Partikularinteressen aus Politik und Wirtschaft (vgl. Weingart 1983, 233).

2 Die Vorgehensweise geht auf Brett Silverstein $(1987,50,54)$ zurück, der einen systematischen Ansatz für Propagandastudien und den Aufbau eines wissenschaftlichen Werkzeugkastens zur Untersuchung der Prozesse und Inhalte von Propaganda forderte. Ein erster Schritt in diese Richtung erfolgte durch das von der internationalen Fachzeitschrift Critical Sociology herausgegebene Symposium zum Thema „Deception, Coercion and Propaganda“. Zwei in dieser Spezialausgabe veröffentlichte Artikel dienen denn auch als Ausgangspunkt für diesen Text (siehe insb. Zollmann 2019 und ebenfalls Bakir et al. 2019). Die kürzlich unter der Leitung englischer und USamerikanischer Forscher gegründete Organisation for Propaganda Studies (http://propagandastudies.ac.uk/about/) soll ebenfalls in diesem Sinne eine historische und gegenwärtige Propagandaforschung fördern. Auch bedankt sich der Autor bei Kolleginnen und Kollegen für wertvolle Anregungen zu einem Vortrag zum Thema auf der 2. Jahrestagung des Netzwerks Kritische Kommunikationswissenschaft 
Es sei noch angemerkt, dass die hier vorgestellte Perspektive auf der kritischen politischen Ökonomie der Massenmedien von Edward S. Herman und Noam Chomsky (2008) basiert und sich damit fundamental von einer sozial-konservativen oder politisch rechten Medienkritik unterscheidet. Herman und Chomsky theoretisieren, wie Massenmedien die Interessen von wirtschaftsliberalen und nationalkonservativen Eliten und damit politisch-ökonomische Ungleichheiten, die zu Lasten der Bevölkerung gehen, unterstützen. So argumentieren Herman und Chomsky (ebd., 1), die Funktion der Massenmedien sei es „,to amuse, entertain, and inform, and to inculcate individuals with the values, beliefs, and codes of behavior that will integrate them into the institutional structures of the larger society. In a world of concentrated wealth and major conflicts of class interest, to fulfil this role requires systematic propaganda." Rechtskonservative Medienkritik fokussiert demgegenüber auf eine vermeintlich liberale Voreingenommenheit der Massenmedien. Demnach seien die Massenmedien dem Staat und der Wirtschaft gegenüber zu feindlich eingestellt oder unterstützten liberale Werte, wie die Gleichstellung der Geschlechter oder Minderheitenschutz (ebd., 196-212). Jüngere Beispiele dafür sind die von US Präsident Donald Trump oder neuen rechten Bewegungen wie der AfD und Pegida gestarteten Kampagnen, die eine oberflächliche Elitenkritik der Massenmedien mit chauvinistischer und patriotischer Rhetorik vermengen. Diese rechte Kritik hat zum Ziel, wirtschaftliche, geschlechtliche oder ethnische Ungleichheiten zu zementieren. Das heißt: die rechte Kritik der Massenmedien repräsentiert nicht die Allgemeinheit, sondern die Interessen des nationalkonservativen Flügels der Eliten. ${ }^{3}$

(„Ideologien \& Ideologiekritik“) an der Ludwig-Maximilians-Universität München sowie bei Uwe Krüger und Sebastian Sevignani für Feedback zu einer früheren Version dieses Textes.

3 Der hier verwendete Ansatz geht davon aus, „dass eine konsensuell geeinte Elite in wichtigen Fragen (Krieg und Frieden, makroökonomische Ordnung) gegen die Interessen eines Großteils der Bevölkerung regieren kann und dass journalistische Eliten zu stark in das Elitenmilieu eingebunden sein könnten, um noch als Anwälte des öffentlichen Interesses kritisch-kontrollierend zu wirken“ (Krüger 2019, 90). Rechte Parteien wie die AfD unterstützen in der Tat die von den bürgerlichen Parteien vorangetriebenen Re-Militarisierungs- und Wirtschaftsliberalisierungsprogramme und sind damit als Teil der konsensual geeinten Elite anzusehen (vgl. Teidelbaum 2016; Winterstein 2019). 


\section{Wie Wissenschaft und Gesellschaft Propaganda verschleiern}

Paul F. Lazarsfeld und Robert K. Merton (1957 [1948], 457-458) hatten bereits in ihrem Klassiker „Mass Communication, Popular Taste and Organised Social Action“ darauf hingewiesen, dass Propaganda in demokratischen Gesellschaften den Platz von direkteren Mitteln der Kontrolle eingenommen habe. Diese Veränderung in der Struktur sozialer Kontrolle verdiene eine gründliche Prüfung, schrieben Lazarsfeld und Merton (ebd., 457-458). Sie verwiesen auf die Massenmedien als eine wichtige Institution der Propaganda, denn diese seien von mächtigen Wirtschaftsinteressen kooptiert worden (ebd., 457-458, 465). Obwohl dieser Text zu den am meist zitierten und kanonisierten Werken in den Medien- und Kommunikationswissenschaften gehört (vgl. Simonson und Weimann 2003, 12), lassen sich in der Fachliteratur kaum Hinweise zu Lazarsfeld und Mertons Bemerkungen zum Thema Propaganda finden.

So ist es in der Tat bemerkenswert, dass in den Medien- und Kommunikationswissenschaften der Zusammenhang von sozialer Kontrolle und Propaganda in westlichen Demokratien bisher kaum untersucht wurde (vgl. Bakir et al. 2019; Zollmann 2019). In den wenigen neueren wissenschaftlichen Abhandlungen zum Thema Propaganda stehen vielmehr die Aktivitäten autoritärer Staaten im Vordergrund. Es findet kaum Berücksichtigung, dass es sich bei den in liberalen Demokratien weitverbreiteten Beeinflussungstechniken, wie PR und Werbung, um Propaganda handeln könnte. Auch werden Journalismus und Nachrichtenmedien strikt von Propaganda abgegrenzt.

Ein Beispiel ist Klaus Mertens Propaganda-Konzeptualisierung: „Anders als die Werbung, setzt Propaganda ihre Intention nicht nur dadurch um, dass sie positive Assoziationen herstellt, sondern in ihrem Anspruch totalitär vorgeht und die Nichtausführung (Nichtbefolgung) der angesonnenen Handlungsalternative mit Sanktionen belegt." (Merten 2000, 150, Hervorhebung im Original) Autoritäre Staaten oder totalitäre politische/religiöse Organisationen, die Sanktionsmittel eher einsetzen als Demokratien und demokratische Organisationen, werden durch diese Sichtweise mit Propaganda in Verbindung gebracht. Merten ignoriert folgenden Sachverhalt: Propaganda wurde insbesondere zu dem Zweck entwickelt, Einstellungen und Handlungsweisen zu beeinflussen, wenn eben nicht auf direkte Sanktionen zurückgegriffen werden kann. Es ist auch 
bemerkenswert, dass Mertens (ebd., 149) Tabelle 2 zum Verständnis von Propaganda unter anderem auf drei Forscher (Lasswell, Smith, Hundhausen) verweist, die in ihren Konzeptionen Werbung und PR als Teil von Propaganda ansahen. Obwohl Tabelle 2 laut Merten "die wesentlichen Strukturelemente" von Propaganda zusammenfasst, grenzt er dann bei seiner eigenen Konzeptualisierung Werbung (Überredung), Propaganda (Manipulation) und PR (Überzeugung) im Prinzip voneinander ab (ebd., $148,151)$. Merten hat somit eine willkürlich eng gefasste Konzeptualisierung vorgenommen, die nicht im Einklang mit wichtigen, von ihm sogar selbst verwendeten, wissenschaftlichen Abhandlungen steht und dazu verleitet, Propaganda von PR und Werbung zu unterscheiden und lediglich auf politische oder religiöse Spezialfälle anzuwenden.

Eine ähnliche Vorgehensweise wählt die Bedeutungsanalyse von Klaus Arnold (2003). Arnold betrachtet zwar das Propaganda Konzept von verschiedenen Seiten und weist darauf hin, „dass der Begriff und das Thema Propaganda in der deutschen Kommunikationswissenschaft in den vergangenen Jahrzehnten kaum beachtet wurden“ (ebd., 63). Auch macht Arnold (ebd.) auf ein Grundlegendes Problem der Wahrnehmung von Propaganda aufmerksam: „Die eigene bzw. die Kommunikation, der man positiv gegenübersteht, wird dabei kaum als Propaganda bezeichnet; Propaganda ist immer das, was der andere, der Gegner macht." Trotz dieser wichtigen Erkenntnisse unterläuft Arnold dann in seiner Analyse von Propaganda der gleiche Fehler, in dem er Propaganda eher autoritären Systemen zuschreibt. So war laut Arnold (ebd., 64) Propaganda in den totalitären Staaten Europas ein „zentrales, die gesamte öffentliche Kommunikation bestimmendes Phänomen“ und Propaganda spiele eine zentrale Rolle „in der öffentlichen Kommunikation der totalitären Staaten“ (ebd., 69). In westlichen Gesellschaften habe sich auf der anderen Seite „die Public Relations mit der Propaganda bzw. mit der Abgrenzung zu ihr beschäftigt“ (ebd., 64). Demnach, so Arnold (ebd.) weiter, hatte sich „die persuasive Kommunikation in den westlichen Systemen professionalisiert" und es musste „eine Distanzierung von ähnlichen, aber negativ bewerteten Kommunikationsformen erfolgen“. Bedeutete die Professionalisierung der Persuasion eine Weiterentwicklung von Propagandatechniken? Stellt Propaganda heute auch in liberalen Demokratien ein zentrales Phänomen dar? Arnold macht zunächst interessante Bemerkungen zu diesen Zusammenhängen: So sei mit dem Eintritt der USA in den Zweiten Weltkrieg Propaganda „als ein Instrument gesehen“ worden, „das auch 
in bzw. von demokratischen Gesellschaften eingesetzt werden kann und muss“ (ebd., 66). Außerdem sei es „die Aufgabe der neuen Disziplin Kommunikationswissenschaft" geworden, "gesichertes Wissen über massenkommunikative Persuasionstechniken zu erarbeiten und den politischen Führern zur Verfügung zu stellen“ (ebd.). Arnold führt diese Sachverhalte dann aber nicht weiter aus; vielmehr grenzt er, wie Merten, PR und Werbung von Propaganda ab (ebd. 74-79). Auch übergeht Arnold die englischsprachige Fachliteratur, die die Entstehung eines enormen Propagandaapparates in den USA, sowie die Rolle, die die Medien- und Kommunikationswissenschaften bei der Verfeinerung von Propagandatechniken spielten, skizziert (vgl. Simpson 1995 für einen Überblick).

Eine seltene Ausnahme in den deutschsprachigen Medien- und Kommunikationswissenschaften ist Michael Kunczik (2010, 39), der bemerkt: „Die Versuche, PR und Propaganda zu unterscheiden, sind allerdings lediglich semantische Spielereien." Und weiter: „Wenn Harold D. Lasswell (1902-1978) Propaganda als die Manipulation von Symbolen definiert, um Einstellungen bezüglich kontroverser Themen zu beeinflussen, dann ist der Begriff Propaganda ohne weiteres ersetzbar durch PR." Wie Kunczik zeigt, wird das allerdings in der Wissenschaft meist nicht so gesehen. „Nach dem Verständnis vieler Autoren (und dem Selbstverständnis vieler PR-Praktiker) unterscheidet sich PR jedoch prinzipiell sowohl von Werbung als auch von Propaganda", konstatiert Kunczik (ebd., 36), und so werde „in vielen Lehrbüchern eine berufsideologisch überhöhte Vorstellung von PR verbreitet" (ebd., 47).

Diese in der Wissenschaft übliche ideologische Betrachtungsweise führt dazu, dass Propaganda eher in einen historischen Zusammenhang gestellt wird, oftmals mit Blick auf den Nationalsozialismus oder Stalinismus. Wenn sich der wissenschaftliche Fokus auf westliche Staaten richtet, dann werden Spezialfälle, wie beispielsweise Propagandakampagnen von Kriegsparteien und Geheimdiensten sowie von politischen oder religiösen Sekten thematisiert. ${ }^{4}$ Demnach ist Propaganda eher ein Problem

Bussemer (2005, 392-393) schreibt, Propaganda bilde heute „nur noch im Spezialbereich der Kriegs- und Kriegsbegründungspropaganda ein öffentlich diskutiertes kontroverses Thema“, welches „,keinerlei gesamtgesellschaftliche Implikationen mehr nach sich zieht". Der Fokus auf Sekten findet sich in der Abhandlung von Merten (2000, 143). Auch aus Arnolds (2003, 63, 73, 78-79) Text lässt sich ableiten, dass Propaganda heute im westlichen Kontext eher auf Spezialfälle im Bereich des Politiksystems (wie Kriegs- und Krisenkommunikation oder Wahlkämpfe) anzuwenden ist. 
vergangener Tage, das im Zeitalter ausdifferenzierter Demokratien und formal unabhängiger Massenmedien an Relevanz verloren hat.

So spricht Thymian Bussemer $(2005,393)$ in der Schlussbetrachtung seiner wissenschaftshistorischen Analyse von einem „Bedeutungsverlust" der Propaganda. Demnach konfrontierten ein dezentralisiertes Mediensystem „hochmoderner oder postindustrieller Gesellschaften“ Propaganda „mit einem schwerwiegenden Reichweiten- bzw. Durchdringungsproblem“, und das "moderne Medienpublikum“ sei in „unzählige Interessens- und Anspruchsgruppen aufgespalten“ (2005, 393). Auch sieht Bussemer in der Pressekonzentration ein Problem des 19. Jahrhunderts und ist der Auffassung, vor allem totalitäre Systeme hätten in der Vergangenheit von zentralisierten Medienstrukturen profitiert $(2005,393)$. Bussemer kann nur deshalb von einem Bedeutungsverlust von Propaganda sprechen, weil er eine Vielzahl von theoretischen und empirischen Forschungsarbeiten bagatellisiert, die die extreme Zentralisierung der Massenmedien in liberalen Demokratien beleuchten und den damit verbundenen Machtmissbrauch auf verschiedenen Ebenen untersuchen (diese Literatur wird in Zollmann 2017, Kapitel 2, besprochen). Auch ignoriert Bussemer die enormen Ressourcen, die Großkonzerne einsetzen, um Propaganda Kampagnen durchzuführen sowie die Fachliteratur zu eben diesen Kampagnen (siehe hierzu Beder 2002; Fones-Wolf 1994; Stauber and Rampton 2004).

In den vergangenen Jahren rückten zwar die Sozialen Medien in den Fokus von Analysen zu untergeordneten Feldern der Propagandaforschung, wie „Fake News“ und Desinformation. Aber auch hier konzentriert sich die wissenschaftliche Aufmerksamkeit in erster Linie auf russische Staatspropaganda oder auf durch Einzelakteure durchgeführte gezielte Manipulation mittels Sozialer Medien. W. Lance Bennett und Steven Livingston (2018) stellen beispielsweise die Desinformationskampagnen der Neuen Rechten und Russlands (insbesondere während des USPräsidentschaftswahlkampfes von 2016) in den Fokus ihrer theoretischen Intervention. Bennett und Livingston vernachlässigen jedoch die Tatsache, dass Desinformationskampagnen in den USA eine lange Tradition haben. Propaganda wurde insbesondere eingesetzt, um die einheimische Bevölkerung von der "Unternehmerfreiheit" zu überzeugen und um die öffentliche Meinung in Europa und der "Dritten Welt" im Sinne von USInteressen zu steuern (siehe hierzu Fones-Wolf 1994; Simpson 1995). Jede 
US-Wahl wurde des Weiteren von enormen Propagandakampagnen begleitet, wobei die größten Investitionen in Wahlkampagnen und Präsidentschaftskandidaten und Kandidatinnen von US-Großkonzernen getätigt werden (siehe hierzu Ferguson et al. 2018).

Generell kann daher festgestellt werden: In den Medien- und Kommunikationswissenschaften wird Propaganda selektiv bearbeitet. Ganz nach dem Motto: Propaganda tritt selten in liberalen Demokratien auf und ist ein Phänomen, das eher autoritären Gesellschaften, Feindstaaten oder dem frühen 20. Jahrhundert zugeordnet werden kann (Bakir et al. 2019, 315). Damit vermeidet der wissenschaftliche Diskurs eine tiefergehende Analyse von Propaganda in westlichen Demokratien. Es kann daher mit Verweis auf Peter Weingart $(1983,228)$ von einer Politisierung der Propagandaforschung gesprochen werden. Denn die Vernachlässigung von Propaganda ist den wirtschaftlichen, staatlichen und nicht-staatlichen Partikularinteressen dienlich, die auch in Demokratien als Propagandaagenturen auftreten.

Im Gegensatz zu dieser Herangehensweise finden sich insbesondere in der frühen Literatur zum Thema einschlägige Hinweise zur Bedeutung von Propaganda in liberalen Demokratien. So schrieb Edward Bernays (2005 [1928], 47-48) im Standardwerk Propaganda, die gegenwärtige Struktur der Gesellschaft mache die Ausübung von Propaganda unvermeidbar. Demnach werde Propaganda in allen wichtigen gesellschaftlichen Teilbereichen von Spezialisten eingesetzt, um die Meinungen von Mehrheiten in die richtigen Bahnen zu lenken (ebd., 47-48, 63).

Der Propagandaforscher Alex Carey $(1995,18)$ bemerkte dazu, dass in westlichen Staaten mehr formale Demokratie und Freiheit mit einem Anstieg von Propaganda einher gingen. Demnach hätten insbesondere die in der USA ansässigen Großkonzerne den Ausbau des Wahlrechts zu Beginn des 20. Jahrhunderts und die Verbreitung des Gewerkschaftswesens als Gefahren für ihre Macht angesehen (ebd., 18-19). Der Ausbreitung der Demokratie, so Carey (ebd., 18), sei dann durch das Verbreiten von professioneller Propaganda entgegengewirkt worden. In demokratischen Gesellschaften könnten Privilegien nicht durch Zwangsmaßnahmen aufrechterhalten werden, schrieb Carey (ebd., 11-17, 21) weiter, daher werde Propaganda als Methode zur Herstellung von Konsens sowie zur sozialen und ideologischen Kontrolle eingesetzt. Careys Analyse legt den folgenden Schluss nahe: Je größer die sozialen Ungleichheiten innerhalb einer auf Klassenstrukturen basierenden demokratischen Gesellschaft sind, 
desto bedeutender wird Propaganda als Instrument zur Sicherung von Macht, Einfluss und Wohlstand.

Studien aus den 1940er und 1950er Jahren belegen in der Tat, dass psychologische und sozialwissenschaftliche Methoden zur Beeinflussung von Meinungen und zur Verhaltenskontrolle am stärksten in jenen Gesellschaften zur Anwendung kommen, die die längste Erfahrung mit liberalen, demokratischen Institutionen haben (vgl. Carey ebd., 12-13). Carey $(1995,12)$ argumentierte wie folgt: „Contrary to common assumptions, propaganda plays an important role - and certainly a more covert and sophisticated role - in technologically advanced democratic societies, where the maintenance of the existing power and privileges are vulnerable to public opinion. In contrast, under authoritarian regimes power and privilege are not open and vulnerable to dissenting public opinion."

Bernays und andere Wirtschaftspraktiker hatten den Begriff Propaganda für eine Bandbreite an Beeinflussungstechniken vorgesehen, die insbesondere in der Produktwerbung, aber auch in anderen Bereichen wie Staatsführung, Organisationskommunikation und Massenmedien erfolgreich eingesetzt wurden (Bernays 2005 [1928]; siehe dazu auch Beder 2002, 121; Carey 1995; Miller und Dinan 2008). Nachdem diese Propagandatechniken von den Kriegsparteien in den beiden Weltkriegen zur Meinungsmanipulation missbraucht worden waren, führten Wirtschaftsleute neue Terminologien ein, insbesondere den Begriff Public Relations (PR) (Miller und Dinan, 2008: 18). Techniken, die in der ersten Hälfte des 20. Jahrhunderts unter dem Propagandalabel zusammengefasst wurden, werden heute PR, Werbung, Branding, Sponsoring, Unternehmenskommunikation, Persuasion, Lobbying, Information Operation (IO), oder Psychological Warfare (Psy-Ops) genannt (siehe dazu auch Miller und Robinson 2019). Damit fand ein Re-Branding von Propaganda statt, das selbst als Teil einer einschlägigen Verschleierungskampagne angesehen werden muss. Denn heute wird die neue Terminologie nicht nur in Wirtschaft, Staat und Militär verwendet, sondern findet ihren Niederschlag auch in vielen Hochschulfächern innerhalb der Human- und Sozialwissenschaften, die sogenannte "persuasive“ Kommunikationstechniken erforschen und unterrichten (vgl. Bakir et al. 2019; Carey 1995; Zollmann 2019). Das bedeutet: In liberalen Gesellschaften werden Techniken der Propaganda bis heute in industriellem Maßstab angewendet, studiert und wissenschaftlich verfeinert. Dies geschieht allerdings unter Verwendung positiv 
konnotierter Begrifflichkeiten und Annahmen, was die Nutzung dieser Techniken angeht (vgl. Carey 1995).

Da Propaganda zu einem wesentlichen Teil auf der Verbreitung von Symbolen beruht, kommt den Massenmedien verschiedener Epochen schon immer eine große Bedeutung zu (vgl. Zollmann 2019). Die Propagandaforscher Garth S. Jowett und Victoria O'Donnell (1992, viii) sehen denn auch die Vielfalt von Informationskanälen als offensichtliche Gelegenheit für vermehrte Propagandaaktivitäten; und obwohl die Massenmedien immer noch als Hauptquelle für Informationsvermittlung in liberalen Demokratien fungieren (vgl. Zollmann 2017), ist es in den Medienund Kommunikationswissenschaften ein "No-Go“, die Massenmedien in den Kontext von Propaganda zu stellen. Die terminologische Verschleierung von Propaganda in Forschung und Gesellschaft sollte allerdings nicht davon abhalten, einen Paradigmenwechsel anzustreben mit dem Ziel, relevante Problemstellungen mit Hilfe einer Propagandaperspektive zu untersuchen.

\section{Propaganda und Massenmedien}

Lediglich das von Herman und Chomsky 1988 etablierte Propagandamodell (PM) analysiert die Überschneidungen von Massenmedien und Propaganda (2008). Das PM wurde in den letzten Jahrzehnten von einer beträchtlichen Anzahl von Forscherinnen und Forschern sowie Studierenden verwendet und weiterentwickelt (vgl. Pedro-Carañana et al. 2018). Die vom PM theoretisierten Prozesse und Hypothesen wurden in zahlreichen Studien empirisch belegt (vgl. Zollmann 2017). Im Vergleich zu ähnlichen Ansätzen, wie zum Beispiel Indexing (Bennett 1990) und Framing (Entman 2004), bleibt das PM allerdings in den Medien- und Kommunikationswissenschaften marginalisiert. Dies hängt mit Hermans und Chomskys Fokus auf durch Massenmedien verbreitete Propaganda zusammen und mit ihrer Vorgehensweise, die Motive der US-Außenpolitik zu hinterfragen. Auch haben Herman und Chomsky keine Operationalisierung von Propaganda vorgenommen (vgl. Boyd-Barrett 2010, 32). Im Folgenden wird daher ein auf die Massenmedien anwendbares Propaganda-Konzept entwickelt.

Propaganda wird oftmals als intentionale Manipulation der öffentlichen Meinung verstanden (vgl. Bussemer 2005, 28; Jowett and O'Donnell 
1992, 4; Silverstein 1987, 51). Nach dieser Lesart stellen das Vorhandensein einer Absicht und eines Agenten bzw. einer Agentur sowie Meinungsmanipulation notwendige Bedingungen von Propaganda dar. Dieser Tatbestand ist auch häufig erfüllt, wenn Propaganda zur Anwendung kommt. Allerdings handelt es sich hierbei nur um einen von verschiedenen Typen (vgl. Ellul 1973, 79). Nach Angaben von Silverstein $(1987,51)$ wird Propaganda auf verschiedene Weisen verbreitet, die intentionale Desinformation und viele andere, subtilere Formen beinhalteten. Bussemer $(2005,33)$ verweist in seiner Studie zu Propaganda auf vielfältige theoretische Konzeptualisierungen. Demnach gebe es eine Lesart, die Propaganda als Primärorgan der gesellschaftlichen Integration verstehe (Bussemer ebd., 34). Diese Propaganda könne zum einen systematisch, von gesellschaftlichen Institutionen ausgehen. Zum anderen könne sie als spontane und unbewusste Verbreitung von Ideologie durch Gesellschaftsmitglieder verstanden werden (ebd.). Demnach diffundiert Propaganda auch innerhalb und zwischen gesellschaftlichen Institutionen und muss nicht zwangsläufig intentional verbreitet werden. Andere Forscher sprechen in diesem $\mathrm{Zu}-$ sammenhang von Integrationspropaganda, die von einer intentionalen Propaganda abzugrenzen ist (vgl. Ellul 1973, 74; Silverstein 1987, 50).

Diese Konzeptualisierung von Propaganda ist im Einklang mit massenmedial verbreiteter Propaganda, die nicht auf intentionalem Verhalten des Agenten/der Agentur (Journalist, Praktiker/Medienorganisation) basieren muss. Allerdings erscheint es am sinnvollsten, von einem Kontinuum zwischen nicht-intentionaler und intentionaler Propaganda zu sprechen. Denn das PM theoretisiert, wie Markt-, Eigentums- und Finanzzwänge als strukturelle Filter auf die Massenmedien einwirken. Gleichzeitig werden diese Filter von Akteuren und Gruppierungen mit Spezialinteressen beeinflusst. $\mathrm{Zu}$ diesen Gruppierungen gehören Medieneigentümer, Direktorien, Werbetreibende, Anteilseigner, Lobbygruppen, Think-Tanks, Netzwerke, Geheimdienste und Politiker (insbesondere Regierungen und Abgeordnete). Diese Akteure haben die Absicht, die Massenmedien im Hinblick auf ihre wirtschaftlichen und politischen Ziele zu manipulieren (vgl. Herman and Chomsky 2008; Miller and Dinan 2008, 4; Zollmann, 2019). Wie die Gatekeeper-Studien belegen, sind Medienorganisationen in ihrem Handlungsspielraum durch die oben genannten Zwänge und Akteure eingeschränkt (vgl. Bennett 1990; Boyd-Barrett 2010; Breed 1955; Gans 1980). Medienschaffende werden in hierarchisch struk- 
turierten Redaktionen sozialisiert und internalisieren dominante Nachrichtenwerte, Normen und Praktiken. Als Resultat passt sich die Medienberichterstattung den ideologischen Erklärungsmustern der Partikularinteressen, die auf die Massenmedien einwirken, an. In diesem Sinne finden wir ein prozesshaftes Zusammenspiel von nicht-intentionaler und intentionaler Propaganda in den Massenmedien (vgl. Zollmann 2017, 2019). Auf der einen Seite wird Propaganda durch Medienschaffende, die institutionellen Zwängen ausgesetzt sind, nicht-intentional produziert und verbreitet. Auf der anderen Seite wirken Medieneigentümer, Politiker oder Lobbyisten absichtlich (intentional) auf die Massenmedien ein, um Propaganda zu erzeugen.

Eine Definition von Propaganda sollte daher zwischen der Verbreitung von Propaganda (die auch nicht-intentional vonstattengehen kann) und den Institutionen und Akteuren, die die Medienlandschaft kontrollieren (und die Massenmedien auch gezielt beeinflussen), unterscheiden. Diese Abgrenzung verlangt auch, zwischen massenmedial und nichtmassenmedial verursachter Propaganda zu differenzieren, wobei die letztere Kategorie auch Maßnahmen wie Lobbyarbeit, Flak oder Staatszensur beinhalten kann.

Demnach wird hier folgende Definition verwendet: „Propaganda ist die Formierung von Texten und/oder Meinungen zugunsten von Partikularinteressen sowie durch massenmedial und/oder nicht-massenmediale Mittel mit dem Ziel, öffentliche Unterstützung und/oder relevantes Handeln zu erreichen. ${ }^{5}$ Diese Definition ist relativ breit angelegt und lässt sich auf die Massenmedien (hier verstanden als massenmedial vermittelte Formierung), die Aktivitäten einer Reihe anderer Institutionen und Akteure (hier verstanden als nicht-massenmedial vermittelte Formierung) und die Rezipienten (hier verstanden als Medienwirkung im Sinne von erreichter öffentlicher Unterstützung und/oder relevantem Handeln) anwenden.

Allerdings sollte eine weitere Qualifizierung getätigt werden. In der Fachliteratur gibt es unterschiedliche Meinungen dazu, ob Propaganda immer als gesellschaftsschädigend anzusehen ist (vgl. Bakir et al. 2019).

5 Die Definition wurde insbesondere unter Berücksichtigung der Konzepte von Bussemer (2005, 29-30), Elter (2005) und Miller und Dinan (2008) entwickelt (vgl. Zollmann 2019, 334-336). 
In ihrem konzeptuellen Framework der „Organised Persuasive Communication (OPC)" unterscheiden Vian Bakir et al. (ebd., 320-325) daher zwischen einem Bündel von Aktivitäten, das konsensuale (gutartige) von nicht-konsensualen (bösartige) Formen der Persuasion unterscheidet. Demnach basierten konsensuale Formen der Persuasion auf einvernehmlichem Dialog, Informationsvermittlung oder einvernehmlicher Überzeugung (ebd., 320-321). Nicht-konsensuale Formen basierten auf Täuschung (Persuasion basierend auf unzureichenden Angaben), Anreizwirkung (Persuasion basierend auf dem Versprechen von Nutzen) und/oder Zwangsmaßnahmen (Persuasion basierend auf einer Drohung mit Sanktionen) (ebd., 321-325). Bakir et al. (ebd., 322) sehen Propaganda als Teil der nicht-konsensualen Persuasion, insbesondere der Täuschung, die wie folgt definiert werden kann: „[...] persuasion via lying, distortion, omission or misdirection. [...] Deceptive information management is non-consensual because it violates the requirement of informed consent; the target of persuasion is unable to reach an informed decision because of inadequate information" [Hervorhebung im Original].

Diese Unterscheidung erlaubt es, die Massenmedien auf Elemente der Täuschung hin zu untersuchen. In den Diskursen der Massenmedien (und speziell im Journalismus) kann Propaganda demnach als Lüge, Verzerrung, Auslassung und/oder Fehlleitung identifiziert werden. ${ }^{6}$ Der Verdacht der Propaganda erhärtet sich, wenn sich auf Täuschung basierende Medieninhalte mit der Ideologie von Partikularinteressen decken. Wenn dies der Fall sein sollte, dann hat das gravierende politische Auswirkungen, denn die den Massenmedien zugeschriebenen normativen Funktionen, wie zum Beispiel die objektive Informationsvermittlung im Falle des Journalismus, sind mit Propaganda nicht $\mathrm{zu}$ vereinbaren.

Im Folgenden wird die auf einer dominanten Ideologie basierende Integrationspropaganda als eine Dimension massenmedialer Propaganda herausgestellt und anhand kurzer Beispiele erläutert. Das Augenmerk richtet sich hier insbesondere auf Propaganda, die in liberalen Qualitätsmedien verbreitet wird. Diese Medien agieren als "Agenda-Setter" und werden insbesondere von Eliten mit Entscheidungsmacht konsumiert (vgl. Zollmann 2017, 62-64). Propaganda in Qualitätsmedien beruht auf einer Auslassung, Verzerrung und Fehlleitung bestimmter Sichtweisen,

$6 \quad$ Der hier vorgeschlagene Bezugsrahmen kann auch auf andere Bereiche wie Werbung oder PR angewendet werden. 
Perspektiven und Fakten. Journalisten lügen in der Regel nicht. Sollten Lügen von Journalisten verbreitet werden, dann eher als Resultat einer professionellen Verwendung von Zitaten offizieller Quellen aus der Politik. Journalisten sind in ihrer Rolle als Informationsvermittler dafür anfällig, auf Täuschung basierende Konstrukte weiterzuverbreiten. Denn insbesondere in liberalen Demokratien greifen Politiker auf Lügen zurück, wenn das dem „nationalen Interesse“ dienlich ist (vgl. Mearsheimer 2011; Miller und Robinson 2019). Das hier skizzierte Bild von Propaganda hat demnach nicht viel mit der Vorstellung einer kruden „Lügenpresse“ zu tun. Vielmehr kennzeichnet sich Propaganda in den Massenmedien durch die Hervorhebung oder Bagatellisierung bestimmter Ideologien und Sachverhalte sowie durch eine selektive Verwendung oder Verzerrung von Fakten aus.

\section{Die Massenmedien als Institution der ideologischen Integration}

Verschiedene Forscher sehen eine Überschneidung von Propaganda und ideologischer Indoktrination (vgl. Ellul 1973, 63-64; Jowett and O'Donnell 1992, 1). Mit Ideologien sind hier interessengeleitete Wertvorstellungen, Bedeutungszuordnungen oder Praktiken von Institutionen bzw. Gesellschaftsgruppen gemeint (vgl. Hall 1977; Philo und Berry 2011, 174). Sollten die Massenmedien die Ideologie einer bestimmten Institution oder Gruppierung überrepräsentieren, dann kann von Integrationspropaganda gesprochen werden. ${ }^{7}$ Integrationspropaganda basiert insbesondere auf der Aufwertung einer Ideologie sowie der Auslassung oder Verzerrung von alternativen Ideologien. Dies führt dazu, dass bestimmte Wertvorstellungen darüber, wie eine Gesellschaft organisiert, in welchem Verhältnis Gesellschaftsmitglieder zueinanderstehen oder wie Politik gemacht werden sollte, eine weite Verbreitung finden. Solche dominanten Ideologien manifestieren sich in verschiedenen gesellschaftlichen Teilsystemen wie Staat, Wirtschaft oder Familie (ausgewählte Beispiele werden

$7 \quad$ Hierbei handelt es sich lediglich um einen von verschiedenen Typen der Propaganda (siehe auch Abschnitt 4). Weitere Typen wie auf Fakten oder Dämonisierung basierende Propaganda werden in Zollmann (2019, 338-341) konzeptualisiert. 
weiter unten in diesem Kapitel veranschaulicht). Durch die Vermittlung dominanter Ideologien üben die Massenmedien eine systemstabilisierende Funktion aus und legitimieren damit gesellschaftliche Machtstrukturen (vgl. Lazarsfeld and Merton 1957 [1948], 465).

Liberale Demokratien befinden sich in einer fortgeschrittenen Phase des Monopolkapitalismus, in der staatliche und wirtschaftliche Machtzentren verzahnt sind (vgl. Foster 2016). Multinational agierende Großkonzerne sind treibende Kräfte in modernen liberalen Demokratien und in der Lage, einen signifikanten Einfluss auf die Politik auszuüben (ebd.). ${ }^{8}$ Integrationspropaganda dient daher auch der Rechtfertigung diesem von der Politik garantiertem Vorrang der Großkonzerne, der nicht demokratisch legitimiert ist. Diesbezüglich argumentieren Jowett und O'Donnell $(1992,214)$, ein Zweck von Propaganda sei „to maintain the legitimacy of the institution or organisation that it represents and thereby ensure the legitimacy of its activities". Es sei noch angemerkt, dass sich die verschiedenen Ausprägungen der Integrationspropaganda je nach Geltungsbereich unterscheiden können. So werden beispielsweise Gewerkschaften in den USA mit großem Aufwand mittels Integrationspropaganda abgewertet. In europäischen Staaten, mit einem ausgeprägteren Sozialwesen ist das nicht unbedingt der Fall. Dort werden Gewerkschaften eher akzeptiert, allerdings wird ihnen ein genauer Platz zu gewiesen, der innerhalb des allgemeinen Rahmens des real existierenden Kapitalismus bleibt.

Es lassen sich verschiedene Schichten der Integrationspropaganda herausarbeiten, die im Folgenden exemplarisch und generalisiert dargelegt werden. Auf einer allgemeinen Ebene sind die Massenmedien ein Stabilisator des real existierenden Kapitalismus (vgl. Curran und Seaton 2003, 103). Institutionen wie Privat- und Konzernunternehmen, Profitmaximierung, das Recht auf Privateigentum, Wirtschaftswachstum oder "freie" Marktwirtschaft werden mit positiven Symbolen belegt und nicht

8 Sharon Beder $(2002,277)$ beschreibt diesen Prozess wie folgt: „Corporate Power has various dimensions. Traditionally, it has been institutionalized in government decision-making structures as a result of the importance of corporate investment to economic growth and the provision of employment. Individual companies can threaten to withdraw that investment if they do not get their way. It is therefore in the interest of government to negotiate and consult extensively with corporate representatives on all policy matters that may affect them. This gives corporations privileged access to government policy-making." 
substanziell hinterfragt (vgl. ebd.). Die dem real existierenden Kapitalismus zugrundeliegende Klassenstruktur, die eine radikale Ungleichheit von Macht, Ressourcen und Möglichkeiten mit sich bringt, bleibt weitestgehend ausgeklammert (vgl. Zollmann 2009). Gleichzeitig werden politische Bewegungen, Institutionen, Parteien und Staaten, die andere Gesellschaftsmodelle vorschlagen, herabgesetzt oder durch negative Assoziation als Bedrohungen dargestellt (vgl. Carey 1995, 16-17, 29).

Im neoliberalen Zeitalter werden selbst sozialdemokratische Institutionen, die laut wissenschaftlichen Studien im 20. Jahrhundert zur Erhöhung des allgemeinen Wohlstands und Wohlbefinden beigetragen haben, verunglimpft. ${ }^{9}$ Der Wohlfahrtsstaat sowie öffentliche Unternehmen, Einrichtungen und Investitionen, die der Bevölkerung wichtige Dienste zu erschwinglichen Preisen liefern, werden als „ineffizient" ausgegeben. Es wird suggeriert, dass diese Institutionen die „individuelle Freiheit" , „Flexibilität" und "Eigeninitiative“ reduzieren oder die "freie“ Wirtschaft behindern würden (siehe dazu Carey 1995, 16-17; Walter 2017a).

Ideologische Integration erstreckt sich nicht nur auf ökonomische Verhältnisse. Trotz einer rechtlich formalen Gleichstellung der Geschlechter wird durch die Massenmedien ein hegemonisches Weltbild der männlichen Dominanz aufrechterhalten. Die Forschung von Karen Ross zeigt, wie dominante Normen und Wertvorstellung über den Platz, die Rolle und die Lebenswelt von Frauen in der Gesellschaft routinemäßig von den Medien bestätigt werden (Ross 2017, 3). So definieren und produzieren Männer zum Beispiel die Nachrichtenagenda und als Resultat thematisieren Nachrichten oftmals Männer und ihre Handlungen; wenn Frauen in diesem Prozess vorkommen, dann meistens in ihrer Rolle als Ehefrauen, Mütter oder Opfer und nur gelegentlich als Politikerinnen oder Expertinnen (Ross et al. 2016, 824).

Auf einer zweiten Ebene lassen sich Propagandakampagnen identifizieren, die die dominante Ideologie verstärken oder sogar neue ideologische Paradigmen einführen. Hierbei kann es sich um spezifische Programme handeln, die im Interesse dominanter Sektoren durchgeführt werden und dann ihren Niederschlag in den Diskursen der Massenme-

$9 \quad$ Siehe dazu die Studie von Ha-Joon Chang (2008), die die gesellschaftlichen Nachteile neoliberaler, kapitalistischer Institutionen wie Deregulierung, Privatisierung, Freihandel und Privateigentum offenlegt und damit die Propaganda entkräftet. 
dien finden. Dazu zählte zum Beispiel die 2001 im Auftrag des Arbeitgeberverbandes Gesamtmetall gestartete „Initiative Soziale Marktwirtschaft", die in Michael Walters (2017b, 197) Worten „den Boden für eine wirtschafts- und sozialpolitische Neuausrichtung der Bundesrepublik bereiten sollte“. Diese von Eliten aus Politik, Wirtschaft und Medien eingeleitete Initiative war Teil einer großangelegten Propagandawelle der Arbeitgeber, die nicht nur zum Ziel hatte, Akzeptanz für marktwirtschaftliche "Reformen" herbeizuführen, sondern auch ein neues öffentliches Verständnis des Sozialen herzustellen (vgl. Walter 2017b, 197-199; auch Nuernbergk 2009, 169). So gelang es der Kampagne der „Initiative Soziale Marktwirtschaft" nach Ansicht von Christian Nuernbergk (2009, 181), "die Interaktionsbedingungen mit mehreren Medienorganisationen strategisch und effizient zu nutzen und eine Berichterstattung zu ihrem überwiegenden Vorteil zu generieren".

Politisch gesehen fand diese Kampagne ihren Ausdruck in der von Bundeskanzler Gerhard Schröder im Jahr 2003 eingeführten und von den bürgerlichen Parteien weitgehend unterstützten Agenda 2010. Von der Politik wurde die Agenda 2010 als eine "Reform“ der Sozialsysteme dargestellt, eine Sichtweise, die in den Leitmedien "große politische Zustimmung" fand (Nullmeier 2008, 160). In Wirklichkeit handelte es sich bei der Agenda 2010 um reaktionäre Sparpolitik, deren Ausrichtung von der neoliberalen Doktrin entnommen war und die zu einer "Verschärfung der sozialen Schieflage“ und "Ausweitung der (Kinder-)Armut bis in die Mitte der Gesellschaft hinein" führte (Butterwegge 2013a). Die Propaganda verdeckte des Weiteren die Tatsache, dass die deutsche Wirtschaft der Hauptnutznießer der Agenda 2010 war und dass diese ohne Rücksicht auf die Lebensqualität der deutschen Bevölkerung und die Stabilität Deutschlands und der EU durchgeboxt wurde. Christoph Butterwegge $(2013 b, 16)$ beschreibt diesen Sachverhalt wie folgt: „Ökonomisch hat sie den ,Standort D' weiter gestärkt, was die wirtschaftliche Unwucht zwischen der Bundesrepublik und Ländern der südlichen EU-Peripherie (Griechenland, Spanien und Portugal) verschärfte und entscheidend zur dortigen ,Staatsschuldenkrise' beitrug. In sozialer Hinsicht wirkte die Agenda-Politik verheerend, weil sie zu einer bis dahin unvorstellbar krassen Verteilungsschieflage bei den Einkommen und Vermögen führte, von der perspektivisch Gefahren für den inneren Frieden und die Demokratie ausgehen" [Hervorhebung im Original]. 
Ein Paradigmenwechsel wurde von den Eliten fast aller politischer Couleur in den 1990er Jahren im Bereich Außenpolitik angestrebt. Als Reaktion auf die verheerenden Angriffskriege der Nazis hatten die Alliierten gesetzliche Restriktionen kodifiziert, die es Deutschland lediglich erlaubten, seine Armee für Verteidigungszwecke einzusetzen. Nach der deutschen Wiedervereinigung und dem Zerfall der Sowjetunion erhöhte sich der Anspruch deutscher Eliten, eine wichtigere Rolle bei internationalen Militäreinsätzen zu spielen. Die durch den "Zwei-Plus-Vier-Vertrag" erlangte Souveränität erlaubte denn auch eine Neuausrichtung der Außenpolitik (vgl. Brehm et al. 2012). Allerdings musste dafür das in der deutschen Gesellschaft vorherrschende friedens- in ein militärpolitisches Verständnis von Außenpolitik umgewandelt werden. Maybritt Brehm et al. (2012) bemerken dazu: „Im Parteienspektrum der neuen Bundesrepublik wurde dieser Diskurs im Großen und Ganzen zugunsten der Militarisierung entschieden." Im Gegensatz dazu war die deutsche Bevölkerung durch die Erfahrung des Nationalsozialismus weitaus weniger auf Krieg eingestimmt.

Ein in den Medien verbreitetes Set von ideologischen Konstrukten wurde daher herangezogen, um eine aggressivere Ausrichtung der Außenpolitik gegenüber der Bevölkerung zu legitimieren. Dazu gehören die Annahmen, Auslandseinsätze der Bundeswehr würden zur Wahrung von "Demokratie“, "Stabilität", oder "Menschenrechten" durchgeführt sowie der Diskurs, beim „Krieg gegen den Terrorismus“ ginge es um die "Sicherheit" und „Freiheit" des Westens. Was Außenpolitik angeht, belegen zahlreiche Studien, wie die Massenmedien diese von westlichen Staatseliten vorgegebenen Erklärungsmuster reflektieren und als bare Münze nehmen (vgl. Zollmann 2017, Kapitel 2). Diese Propaganda zielt also darauf ab, die Außenpolitik mit positiven Assoziationen zu besetzen, um die Meinungskluft zwischen Eliten und Bevölkerung zu schließen. Uwe Krügers (2019) Studie wirft ein Licht auf diesen Sachverhalt. Krüger $(2019,257)$ belegt, wie die Kommentare und Leitartikel von vier deutschen Alpha-Journalisten mit der Argumentation von Nato- und US-nahen Politiknetzwerken korrelierten. Demnach benutzten die Journalisten unkritisch den "erweiterten Sicherheitsbegriff" und "argumentierten für ein stärkeres militärisches Engagements Deutschlands v.a. in Afghanistan, das von der Nato und den USA gewünscht, von der deutschen Bevölkerung jedoch mehrheitlich abgelehnt wird," so Krüger (ebd.). Auch sei die ablehnende Haltung der Bevölkerung gegenüber Militäreinsätzen zum 
Teil von den Journalisten diffamiert worden und es wurde „der Bundesregierung eine verstärkte Überzeugungsarbeit am Volk empfohlen“ (ebd.).

Integrationspropaganda zur Außenpolitik wird häufig durch eine zentrale, auf Selektivität basierende ideologische Grundannahme unterfüttert: Die von den USA angeführte westliche Staatengemeinschaft nimmt eine positive Rolle in der Welt ein. Eine substanzielle Kritik, die die ökonomischen Ziele der Außenpolitik untersucht und deren Auswirkung moralisch oder juristisch bewertet, erfolgt kaum (vgl. Zollmann 2017, 23-24). Wie freigegebene US-Regierungsdokumente belegen, ist das Primärziel der US-Außenpolitik die Sicherstellung ausländischer Ressourcen und Märkte für die Ausbeutung durch westliche Großkonzerne (vgl. Zollmann 2016, 2017, 217). Die neue Außenpolitik Deutschlands verfolgt die gleichen Ziele, oftmals als Juniorpartner der USA (vgl. Zollmann 2016). Eine auf ruchlosen Motiven wie imperialistischer Machterweiterung basierende Außenpolitik wird in den Nachrichtenmedien allerdings nur sogenannten "Schurkenstaaten“ zugeschrieben, zu denen Russland, Syrien, Iran oder Nordkorea gezählt werden. Der gleiche Bewertungsmaßstab wird nicht auf westliche Staaten angewandt. Integrationspropaganda formiert demnach ein dichotomes Bild, das die Welt in Gut und Böse unterteilt, wobei „wir" die Guten sind (vgl. ebd.).

Dabei verschleiert die Propaganda, dass die USA zwischen 1945 und 2005 versucht haben, mehr als fünfzig ausländische Regierungen zu stürzen und dreißig populär-nationalistische Bewegungen zu zerschlagen, die über die eigenen Ressourcen in ihrem Land bestimmen wollten (vgl. Blum 2006, 1-2). Viele dieser Interventionen richteten sich gegen progressive Regierungen, die den Lebensstandard ihrer Bevölkerungen durch wirkliche Reformen verbessern wollten. Soziale Reformen gehen aber oftmals zulasten des "Investitionsklimas" und damit der Profitinteressen ausländischer Großkonzerne, weil sie mit besseren Lohnbedingungen der Bevölkerung und höheren Unternehmenssteuern einhergehen. William Blum belegt in seinem Buch, dass dies die Hintergründe der USMilitärinterventionen waren (vgl. Blum 2006). Dennoch richten sich die deutschen Leitmedien oftmals nach den Interessen der USA und Nato (vgl. Krüger 2019; Zollmann 2017). 


\section{Schlussbemerkung}

Zunächst wurde aufgezeigt, wie die Wissenschaft und Gesellschaft liberaler Demokratien Propaganda terminologisch verschleiern. Konzepte, die es ermöglichen, massenmedial verbreitete Propaganda zu untersuchen, wurden daher vorgestellt. Insbesondere ideologische Integrationspropaganda, die zur Stabilisierung der Interessen gesellschaftlicher Machtzentren eingesetzt wird, wurde anhand von Beispielen erörtert. Nicht diskutiert wurden demgegenüber andere Aspekte propagandistischer Mediendiskurse, wie die selektive Verwendung von Fakten und parteiische Dämonisierungskampagnen (für eine weiterführende Diskussion verschiedener Typen von Propaganda vgl. Zollmann 2019, 338-341). Der von Forschern konstatierte Verfall demokratischer Institutionen (vgl. Bennett und Livingstone 2018, 127-128, 134), hat seine Ursache auch in Propaganda. Denn die immer größer werdende Kluft zwischen den realen sozialen Verhältnissen und dem durch Propaganda vermittelten Image eben dieser sozialen Verhältnisse kann nicht mehr hinreichend überbrückt werden. Vielen Menschen ist bewusst, dass die bürgerlichen Parteien kaum noch voneinander zu unterscheiden sind. Innenpolitisch wird parteiübergreifend eine Politik des Sozialabbaus betrieben; außenpolitisch haben militaristische Tendenzen die Oberhand gewonnen. Propaganda hat damit zum gefährlichen Erstarken der Neuen Rechten und zur fortschreitenden sozialen Erosion und Apathie beigetragen. Die hier aufgezeigten Sachverhalte zu Propaganda in liberalen Demokratien stellen nur eine erste Annäherung dar. Weitere Untersuchungen zur Rolle massenmedial verbreiteter Propaganda in liberalen Demokratien sind zwingend erforderlich.

\section{Literatur}

Arnold, Klaus. 2003. „Propaganda als ideologische Kommunikation“. Publizistik 48 (1): 63 82.

Bakir, Vian, Eric Herring, David Miller, und Piers Robinson. 2019. „Organised persuasive communication: a new conceptual framework for research on public relations, propaganda and promotional culture". Critical Sociology 45 (3): 311-328.

Beder, Sharon. 2002. Global Spin: the Corporate Assault on Environmentalism. Foxhole: Green Books. 
Bennett, Lance, und Steven Livingston. 2018. „The Disinformation Order: Disruptive Information and the Decline of Democratic Institutions". European Journal of Communication 33 (2): 122-139.

Bennett, W. Lance. 1990. „Toward a Theory of Press-State Relations in the United States”. Journal of Communication 40 (2): 103-125.

Bernays, Edward. 2005 [1928]. Propaganda. New York, NY: IG Publishing.

Blum, William. 2006. Rogue State: a Guide to the World's Only Superpower, 3. Aufl. London: Zed Books Ltd.

Boyd-Barrett, Oliver. 2010. „Recovering agency for the propaganda model: The implications for reporting war and peace". In Peace Journalism, War and Conflict Resolution, herausgegeben von Richard Lance Keeble, John Tulloch, und Florian Zollmann, 31-48. New York, NY: Peter Lang.

Breed, Warren. 1955. „Social control in the newsroom: a functional analysis”. Social Forces 33 (4): 326-335.

Brehm, Maybritt, Koch, Christian, Ruf, Werner, und Peter Strutynski. 2012. Armee im Einsatz - 20 Jahre Auslandseinsätze der Bundeswehr. Eine Bilanz. AG Friedensforschung. http://www.ag-friedensforschung.de/themen/Bundeswehr/armee-im-einsatz.html. Zugegriffen: 14. Juli 2019.

Bussemer, Thymian. 2005. Propaganda: Konzepte und Theorien. Wiesbaden: VS Verlag für Sozialwissenschaften.

Butterwegge, Christoph. 2013a. „Die Agenda 2010 - Begründung und Legitimationsbasis für eine unsoziale Politik". NachDenkSeiten, 13. März. https://www.nachdenkseiten.de/?p=16494\#note_6. Zugegriffen: 12. Juli 2019.

Butterwegge, Christoph. 2013b. Gerhard Schröders Agenda 2010: Zehn Jahre Unsoziale Politik. Analysen. Berlin: Rosa Luxemburg Stiftung. https://www.rosalux.de/filead-

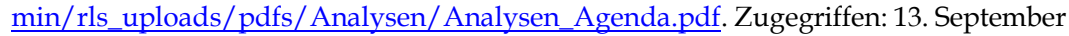
2019.

Carey, Alex. 1995. Taking the Risk Out of Democracy: Corporate Propaganda Versus Freedom and Liberty. Urbana and Chicago, IL: University of Illinois Press.

Chang, Ha-Joon. 2008. Bad Samaritans: the Myth of Free Trade and the Secret History of Capitalism. New York, NY: Bloomsbury Press.

Curran, James, und Jean Seaton. 2003. Power Without Responsibility: the Press, Broadcasting, and New Media in Britain, 6. Aufl. London: Routledge.

Dudley, Drew. 1947. "Molding Public Opinion Through Advertising”. The ANNALS of the American Academy of Political and Social Science 250 (1): 105-112.

Ellul, Jacques. 1973. Propaganda: the Formation of Men's Attitudes. New York, NY: Vintage Books.

Elter, Andreas. 2005. Die Kriegsverkäufer: Geschichte der US-Propaganda 1917-2005. Frankfurt am Main: Suhrkamp.

Entman, Robert M. 2004. Projections of Power: Framing News, Public Opinion, and U.S. Foreign Policy. Chicago, IL: University of Chicago Press. 
Ferguson, Tom, Jorgensen, Paul, und Jie Chen. 2018. „Industrial structure and party competition in an age of hunger games: Donald Trump and the 2016 Presidential Election". Working Paper No. 66, January, 1-100. https://www.ineteconomics.org/uploads/papers/Ferg-Jorg-Chen-INET-Working-Paper-Industrial-Structure-and-PartyCompetition-in-an-Age-of-Hunger-Games-8-Jan-2018.pdf. Zugegriffen: 15. Juli 2019.

Fones-Wolf, Elizabeth A. 1994. Selling Free Enterprise: the Business Assault on Labor and Liberalism. Urbana and Chicago: University of Illinois Press.

Foster, John Bellamy. 2016. „Monopoly Capital at the half-century mark”. Monthly Review 68: 3. https:// monthlyreview.org/2016/07/01/monopoly-capital-at-the-half-centurymark/. Zugegriffen: 30. September 2019.

Gans, Herbert J. 1980. Deciding What's News: a Study of CBS Evening News, NBC Nightly News, Newsweek and Time. London: Constable.

Hall, Stuart. 1977. "Culture, the media and the 'ideological effect'”. In Mass Communication and Society, herausgegeben von James Curran, Michael Gurevitch und Jane Woollacott, 315-348. London: Edward Arnold.

Herman, Edward S., und Noam Chomsky. 2008. Manufacturing Consent. The Political Economy of the Mass Media, 3. Aufl. London: The Bodley Head.

Jowett, Garth S., und Victoria O'Donnell. 1992. Propaganda and Persuasion, 2. Aufl. London: Sage.

Krüger, Uwe. 2019. Meinungsmacht: Der Einfluss von Eliten auf Leitmedien und Alpha-Journalisten - eine kritische Netzwerkanalyse. 2., erweiterte und überarbeitete Auflage. Köln: Herbert von Halem.

Kunczik, Michael. 2010. Public Relations - Konzepte und Theorien. 5., erweiterte und überarbeitete Auflage. Köln, Weimar, Wien: Böhlau.

Lasswell, Harold D. 1971 [1927]. Propaganda Technique in World War I. Cambridge, Mass.: M.I.T. Press.

Lazarsfeld, Paul F., und Robert K. Merton. 1957 [1948]. „Mass communication, popular taste and organised social action". In Mass Culture: the Popular Arts in America, herausgegeben von Bernard Rosenberg und David Manning White, 457-473. New York, NY: The Free Press.

Mearsheimer, John J. 2011. Why Leaders Lie: the Truth about Lying in International Politics. Oxford: Oxford University Press.

Merten, Klaus. 2000. Struktur und Funktion von Propaganda. Publizistik 45 (2): 143-162.

Miller, David, und Piers Robinson. 2019. „Propaganda, Politics and Deception”. In The Palgrave Handbook of Deceptive Communication, herausgegeben von Tony Docan-Morgan, 969-988. Cham: Springer Nature/Palgrave.

Miller, David, und William Dinan. 2008. A Century of Spin: how Public Relations became the Cutting Edge of Corporate Power. London: Pluto Press.

Nuernbergk, Christian. 2009. „Die PR-Kampagne der Initiative Neue Soziale Marktwirtschaft und ihr Erfolg in den Medien: Erste Ergebnisse einer Evaluationsstudie“". In PRKampagnen: Über die Inszenierung von Öffentlichkeit, herausgegeben von Ulrike Röttger, 167-184. 4., überarbeitete und erweiterte Auflage. Wiesbaden: VS Verlag für Sozialwissenschaften. 
Nullmeier, Frank. 2008. Die Agenda 2010: Ein Reformpaket und sein kommunikatives Versagen. Bertelsmann Stiftung, Reformkompass. http:/ / www.reformkompass.de/uploads/tx_itao_download/Fallstudie_Agenda_2010_Download_02.pdf. Zugegriffen: 12. Juli 2019.

Pedro-Carañana, Joan, Broudy, Daniel, und Jeffery Klaehn. 2018. The Propaganda Model Today: Filtering Perception and Awareness. London: University of Westminster Press.

Philo, Greg, und Mike Berry. 2011. More Bad News from Israel. London: Pluto Press.

Ross, Karen. 2017. Gender, Politics, News: A Game of Three Sides. West Sussex: John Wiley \& Sons.

Ross, Karen, Boyle, Karen, Carter, Cynthia, und Debbie Ging. 2016. „Women, men and news: It's life, Jim, but not as we know it". Journalism Studies 19 (6): 824-845.

Scheu, Andreas, und Thomas Wiedemann. 2008. „Kommunikationswissenschaft als Gesellschaftskritik: Die Ablehnung linker Theorien in der deutschen Kommunikationswissenschaft am Beispiel Horst Holzer". medien \& zeit 23 (4): 9-17.

Simonson, Peter, und Gabriel Weimann. 2003. „Critical research at Columbia: Lazarsfeld's and Merton's 'Mass communication, popular taste, and organized social action'". In Canonic Texts in Media Research: Are There Any? Should There Be? How About These?, herausgegeben von Elihu Katz, John Durham Peters, Tamar Liebes, und Avril Orloff, 12-38. Cambridge, MA: Polity Press.

Simpson, Christopher. 1995. Science of Coercion: Communication Research E Psychological Warfare, 1945-1960. New York, NY: Open Road.

Silverstein, Brett. 1987. „Toward a science of propaganda”. Political Psychology 8 (1): 49-59.

Stauber, John und Rampton, Sheldon. 2004. Toxic Sludge is Good for You: Lies, Damn Lies and the Public Relations Industry. London: Robinson.

Teidelbaum, Lucius. 2016. „Die AfD als Partei des (alten) deutschen Militarismus. Informationsstelle Militarisierung“. IMI-Analyse Nr. 17, 15. Juni. http://www.imi-online.de/download/IMI-Analyse2016-17-AfdLuciusT.pdf. Zugegriffen: 24. September 2019.

Walter, Michael. 2017a. Reformvisionen: Zur Bildpolitik Wirtschafts- und Sozialpolitischer Reforminitiativen. Köln: Herbert von Halem.

Walter, Michael. 2017b. „Die symbolische Dekonstruktion des Sozialstaates als elitäres PRProjekt“. In Lügen die Medien? Propaganda, Rudeljournalismus und der Kampf um die Öffentliche Meinung, herausgegeben von Jens Wernicke, 196-211. Frankfurt am Main: Westend.

Weingart, Peter. 1983. „Verwissenschaftlichung der Gesellschaft - Politisierung der Wissenschaft". Zeitschrift für Soziologie 12 (3): 225-241.

Winterstein, Richard. 2019. „Die ideologische Integrationsleistung der AfD“. Telepolis, 21.4., https://www.heise.de/tp/features/Die-ideologischen-Integrationsleistungen-derAfD-4399353.html?seite=all. Zugegriffen: 24. September 2019.

Zollmann, Florian. 2019. „Bringing Propaganda Back into News Media Studies”. Critical Sociology 45 (3): 329-345.

Zollmann, Florian. 2017. Media, Propaganda and the Politics of Intervention. New York, NY: Peter Lang. 
Zollmann, Florian. 2016. „Unterm Brennglas... Warum sind wir die Guten?“ Publik-Forum Extra Leben, Februar 2016.

Zollmann, Florian. 2009. „Is it either or? Professional Ideology vs. Corporate-Media Constraints". Westminster Papers in Communication and Culture 6 (2): 97-118.

\section{Open Access}

Dieser Beitrag erscheint unter der Creative-Commons-Lizenz CC-BY-SA 4.0:

https://creativecommons.org/licenses/by-sa/4.0/ 